\title{
TRANSMISSION POLARIZED LIGHT MICROSCOPY OF CARIOUS HUMAN DENTAL ENAMEL
}

\author{
Aline de Almeida Neves*, Frederico Barbosa de Sousa**, Luiz Carlos Pereira*, Laércio Guzela* \\ * Materials Science and Metallurgy Department, Federal University of Rio de Janeiro, Brazil \\ ** Department of Morphology, Federal University of Paraíba, Brazil
}

Dental enamel is a highly mineralized tissue, composed at the ultrastructural level, of crossectioned rhomboidal apatite cristalites $(0,04 \mu \mathrm{m}$ width) tighly packed along prismatic structures in which their long dimensions are more or less paralell with the direction of the enamel prisms [1].

Carious lesions are resulted from acid production in thick biofilm accumulations leading to enamel demineralization following the course of the prisms until the dentinal tissue. Generally, it is a point in caries progression where enamel does not support mechanical efforts, breaking into a cavity [2].

In polarized light dental enamel behaves as an uniaxial body, birefringent in all directions except for the optic axis, if the prisms are paralell to the plane of the section. Total birefringence (BR) is given by the sum of intrinsic (negative) BR resulted from apatite and form (positive) BR, which is given by imbibition in a medium with refractive index different from enamel $(1,62)$. Its magnitude can be obtained by relating the optical path difference between the ordinary and extraordinary ray (retardation) and the thickness of the sample [3]. As poorly mineralized areas possess higher values of form BR, total BR measures can be used in morphologic studies of carious lesions.

Primary molars presenting white spots lesions at approximal surfaces were transversely sectioned (Fig.1) and thin sections $(100 \mu \mathrm{m})$ were produced by grinding in sandpaper and polishing with alumina $(1 \mu \mathrm{m})$. They were mounted imbibed in water $(\mathrm{RI}=1.33)$ and angle of extinction was obtained as a mean of five readings (Fig.2). The stage was rotated $45^{\circ}$ subtracted to the extinction and a Berek compensator was used to measure retardation each $5.5 \mu \mathrm{m}$ along two traverses perpendicular to each other (Fig.3). BR was obtained by dividing retardation by the sample thickness and graphs were plotted for transversal and longitudinal traverses (Graphs 1 and 2).

Examination of BR between points 6 and 8 (Graph 1) reveals that this region behaves like an isotropic body due to the high form birefringence caused by replacement of pores in demineralized enamel by the imbibition medium. However, the hole longitudinal lesion is divided by an area of negative birefringence (points 5 and 6) which can be explained by the course of prisms as seen in Figure 4A. This area was probably not affected by the demineralization because of being located the contact point, where bacterial accumulation is impaired by interdental attrition. The shape of the transversal BR, where the greatest degree of form birefringence in located between points 9 and 12 (Graph2) reveal an area of great demineralization, correlating to the course of prisms in Fig 4B.

\section{References}

[1] H.Warshawsky, Anat. Rec. 224 (1989) 42.

[2] A. Thylstrup et al, Adv. Dent. Res. 8 (1994) 144.

[3] D. Carlström, Adv. Oral. Biol. 1 (1964) 255. 


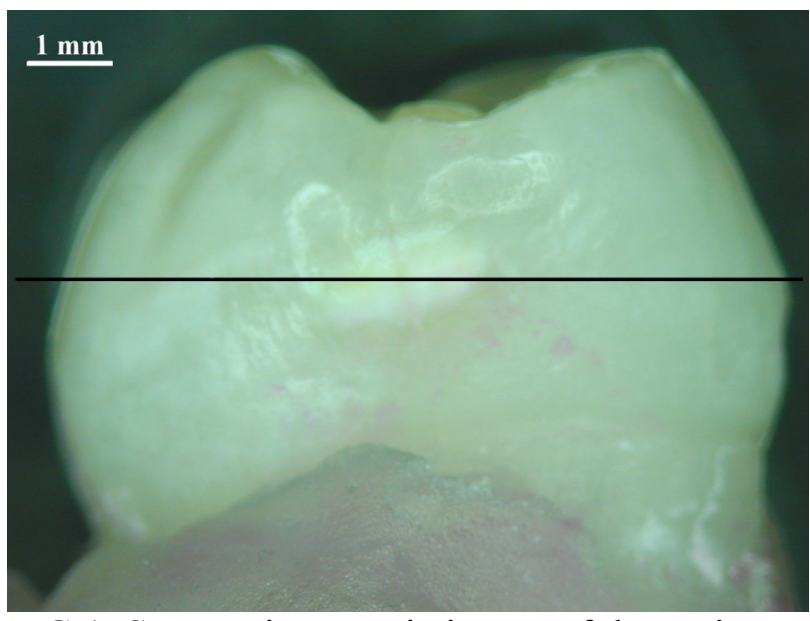

FIG 1. Stereomicroscopic image of the carious lesion. Line indicates location of the ground section.

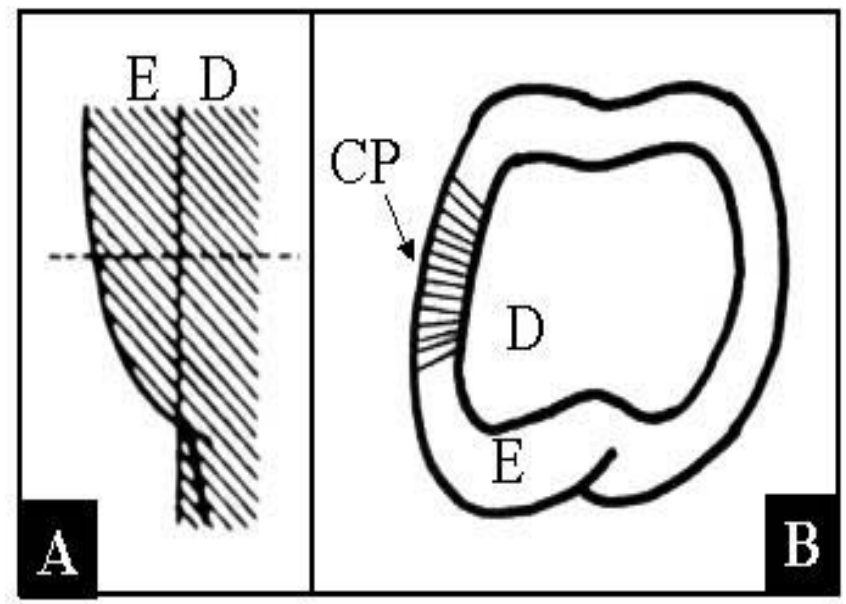

FIG 3. Scheme of the course of the enamel prisms in primary teeth. $\mathrm{E}=$ enamel, $\mathrm{D}=$ dentin, $\mathrm{CP}=$ contact point. A) Longitudinal view. $\mathrm{B}$ ) Transversal view.

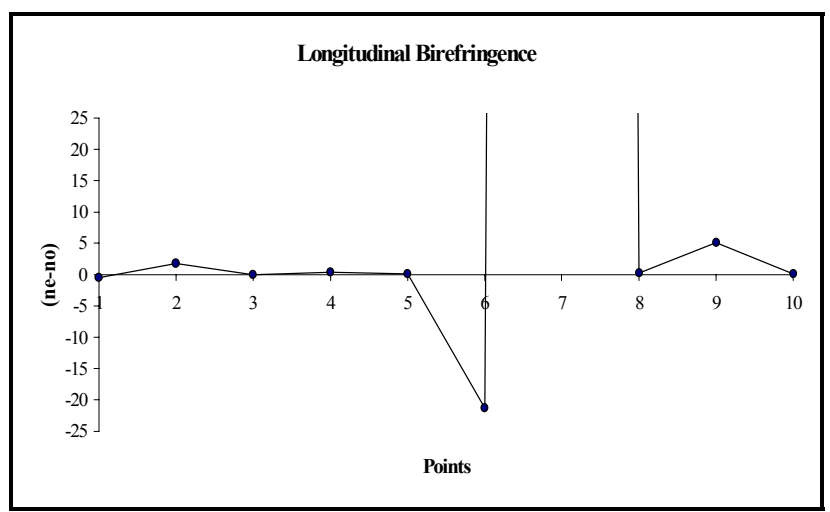

GRAPH 1. Birefringence along the longitudinal traverse with imbibition in water.

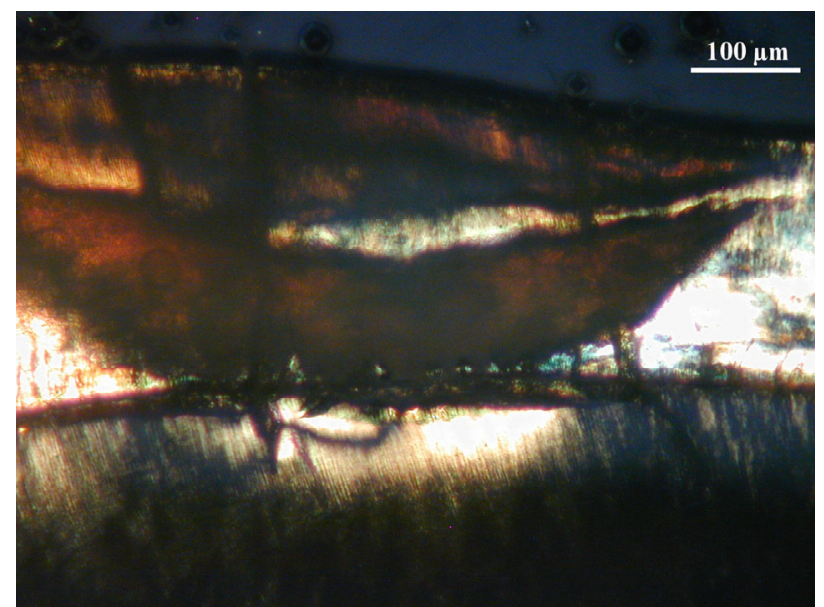

FIG 2. Ground section of carious lesion in polarized light at the extinction position. Note lack of extinction in the bright areas.

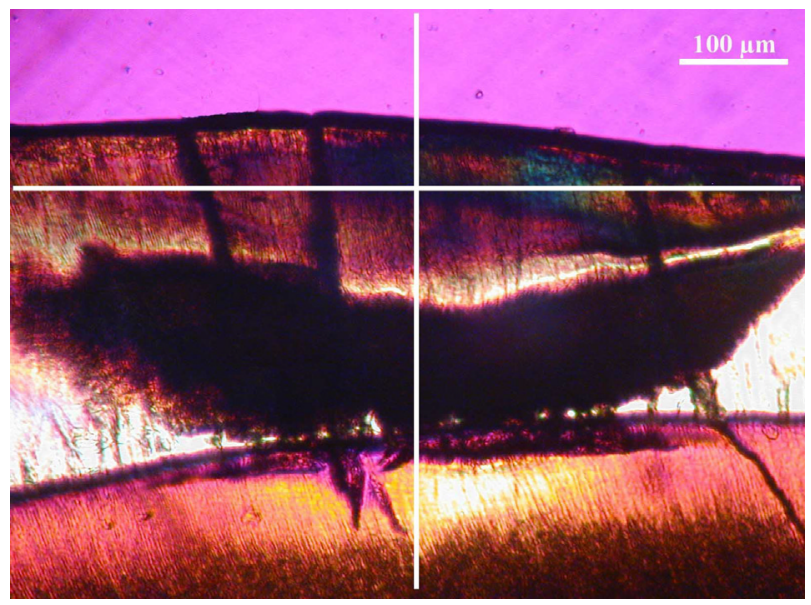

FIG 4. Ground section of carious lesion in polarized light at the subtraction position (sensitive tint plate). Lines indicates path of retardation measures.

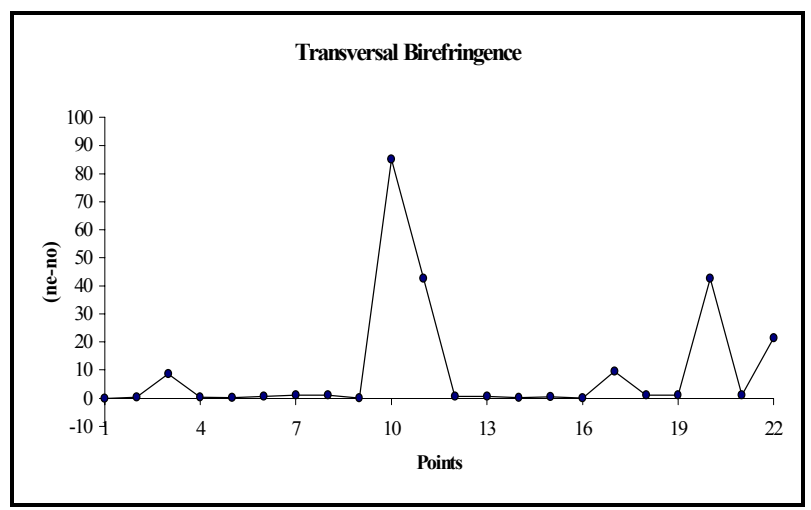

GRAPH 2. Birefringence along the transversal traverse with imbibition in water. 